Shorland F. B., Gerson I., Hansen R. P. The branched-chain fatty acids of butterfat. VI. Investigation of the $\mathrm{C}_{13}$ acids. Biochem. J., 1955b, 61, 702 .

Sмгтн L. M. Quantitative fatty acid analysis of milk fat by gas liquid ehromatography. J. Dairy Sci., 1961, 44, 607-622.

SмIтн L. M., JACK E. The unsaturated fatty acids of milk fat. III. Geometrical isomerism. $J$. Dairy Sci., 37, 399-406.

Stadhodders J., Mulder H. The composition of dutch butterfat. I. Seasonal variations in the unsaturated fatty acid composition of butterfat. Neth. Milk Dairy J., 1955, 9, 182-193.

Stadhouders J., Mulder H. The composition of dutch butterfat. II. Seasonal variations in the saturated fatty acid composition of butterfat. Neth. Milk Dairy J., 1956, 10, 53-59.

Thompson M. P., Brunner J. R., Stine C. M. Characteristics of high melting triglyceride fractions from the fat globule membrane and butter oil of bovine milk. J. Dairy Sci., 1959, 42, 1651-1658.

WolfF J. P. Application de la chromatographie en phase vapeur au contrôle de la pureté des beurres. Ann. Fals, Exp. Chim., 1960, 53, 318-343.

\title{
VARIATION DE LA TENEUR DU KÉFIR EN VITAMINE B 12 PENDANT SA FABRICATION ET SA MATURATION
} par

B. PANIĆ, J. RAIŠC, V. HRISTIĆ Belgrade (Yougoslavie)

Le Kéfir représente un des laits fermentés, dont l'importance dans la nourriture humaine est grande, en plusieurs pays. Depuis quelque temps, on produit et on fait usage de plus en plus du kéfir en Yougoslavie.

Cependant, on a, relativement, peu de faits sur l'importance nutritive de cette boisson fermentée et, surtout sur sa teneur en vitamine [1]. On sait que des changements remarquables du contenu du lait en éléments particuliers ont lieu pendant la fabrication et la maturation des boissons fermentées, changements qui sont le résultat de l'activité biochimique des ferments lactiques. Ainsi, d'après les résultats de nos recherches [2] et d'après les recherches d'autres auteurs $[3,4,5]$ les laits fermentés, comme on l'a constaté pour le yoghourt, peuvent contenir certaines vitamines en moindre quantité que les produits frais, c'est-à-dire, le lait cuit à partir duquel ils 
sont faits. Cela veut dire que pendant les processus de fabrication et de maturation des boissons fermentées, il peut se produire des pertes, quelquefois importantes, dans la teneur en certaines vitamines. Ce fait représente une donnée très importante pour une connaissance générale et complète de l'importance nutritive de certains produits lactés, non seulement du point de vue scientifique, mais aussi au point de vue pratique.

Dans ce travail nous avons examiné les changements de la teneur en vitamine B 12 pendant la fabrication et la maturation du kéfir, quant à l'importance spéciale de cette vitamine dans l'alimentation humaine.

\section{Expérimentation}

Le Kéfir est fait du lait de vache qu'on a prélevé de laiteries de Belgrade. La technologie de la production du Kéfir était la suivante :

a) Kéfir fait de lait auquel on ajoute les grains du Kéfir.

Les grains de Kéfir, qui sont périodiquement (de 5 à 7 jours) rincés à l'eau stérilisée, sont mis dans du lait refroidi à $25^{\circ} \mathrm{C}$. Le lait était précédemment chauffé à $90-95^{\circ} \mathrm{C}$, pendant une demi-heure. Les grains de Kéfir ainsi arrosés sont incubés pendant 16 à 18 heures à la température de $21-22^{\circ} \mathrm{C}$.

Après le caillage du lait, le Kéfir est filtré sur crible, et les grains du Kéfir qui sont restés sur le crible sont arrosés de nouveau avec le lait qui était traité précédemment par la chaleur. Le Kéfir obtenu est maintenu à une température de $8^{\circ}-10^{\circ} \mathrm{C}$, pendant un à trois jours, pour subir la maturation.

\section{b) Kéfir fait de lait auquel on ajoute la culture-mère.}

On fait cailler le lait par les grains de Kéfir qui servent comme culture-mère pour la production du kéfir dans les conditions industrielles. Le lait qui était précédemment chauffé à $90-95^{\circ} \mathrm{C}$ pendant une demi-heure et refroidi à $25^{\circ} \mathrm{C}$, est inoculé, à $5-7 \mathrm{p}$. 100 , avec la culture-mère. Le lait inoculé est incubé pendant 16 à 18 heures à la température de $21-22^{\circ} \mathrm{C}$. Le Kéfir ainsi obtenu est gardé aussi à $8-10^{\circ} \mathrm{C}$ pendant 1 à 3 jours, pour subir la maturation.

On a dosé la vitamine B12 par la méthode microbiologique avec la souche $E$. coli $113-3$ de la manière suivante : $25 \mathrm{ml}$ de lait ou Kéfir est dilué dans $100 \mathrm{ml}$ d'eau distillée en ajoutant $400 \mu \mathrm{g} \mathrm{KCN}$. En ajoutant quelques gouttes de HCL 10 p. 100, le $p H$ est ajusté entre 4,5-5,0. Les échantillons sont autoclavés 10 minutes à une d'atmosphère. Après l'autoclavage on ajoute aux échantillons de 
lait et de Kéfir un à $10 \mathrm{ml}$ de solution saturée d'acétate de plomb. A quantité fixée de filtrat plus ou moins trouble on ajoute encore $1 \mathrm{ml}$ de la solution saturée de $\mathrm{Na}_{2} \mathrm{SO}_{4}$, après quoi le filtrat est adouci par l'eau distillée, relevant du contenu de la vitamine B12 et le $p \mathrm{H}$ final amené à 7,0 au moyen de $\mathrm{NaOH} 10$ p. 100. Par filtration répétée on obtient un filtrat tout à fait pur, convenable pour la détermination de la vitamine B12 par cette méthode.

\section{Résultat et discussion}

Par les données représentées par le tableau I et les figures 1 et 2, on peut voir que pendant la fabrication et la maturation du Kéfir on a des pertes en vitamine B12; c'est pour cela que le contenu de la vitamine dans ce produit est remarquablement moindre que dans le lait cuit duquel il est préparé. La diminution de la vitamine B12 est surtout rapide pendant la première phase de la production du Kéfir, c'est-à-dire pendant les 18 heures d'incubation du lait ensemencé avec les grains de Kéfir, e'est-à-dire par la culture-mère, à la température de $22^{\circ} \mathrm{C}$. Dans les phases suivantes de la maturation du Kéfir, quand le Kéfir est à la température de $8-10^{\circ} \mathrm{C}$, les pertes sont moindres (tableau I).

Cependant, en comparant les données pour le Kéfir fait du lait auquel on a ajouté les grains du Kéfir avec les données pour le Kéfir fait du lait auquel on a ajouté la culture-mère (le lait qu'on fait cailler par les grains de Kéfir), on constate un fait intéressant : la perte en vitamine n'est pás la même pour les deux sortes de Kéfir. Dans le Kéfir fait par adjonction des grains de kéfir (Figure 1), pendant la maturation à la température de $8^{\circ}$ à $10^{\circ} \mathrm{C}$ la teneur en vitamine $\mathrm{Bl} 2$ augmente un peu, avec maximum à 24 heures, et puis au cours des deuxième et troisième jours elle tombe de nouveau, en atteignant après le troisième jour approximativement la même valeur qu'après la phase d'incubation à la température de $22^{\circ} \mathrm{C}$. Entre les valeurs des deuxième et troisième jours les différences ne sont pas notables bien que l'on note une tendance à une diminution insignifiante du contenu en vitamine B12 au cours du troisième jour.

Dans le Kéfir fait du lait auquel on a ajouté la culture-mère, on constate une tendance un peu différente car la diminution est constante dans toutes les phases successives de la fabrication et de la maturation. Mais, même dans ce kéfir, la plus grande diminution du taux de vitamine B12 est dans le processus de la fabrication, c'est-à-dire, pendant le comportement du lait inoculé à la température de $22^{\circ} \mathrm{C}$. 
TABLEAU I

TENEUR MOYENNE DU KéfiR EN VITAMINE B 12 PENDANT LES PHASES PARTICULIÈRES DE LA FABRICATION

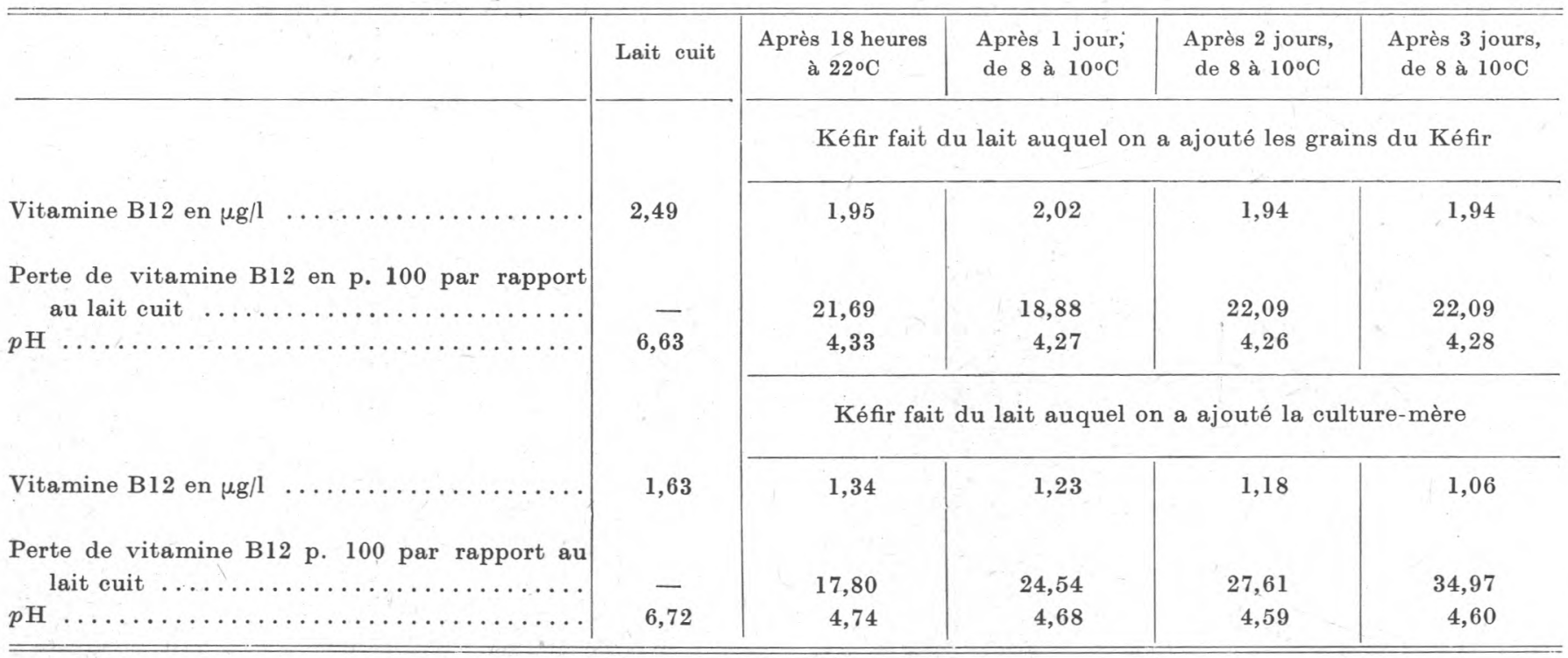




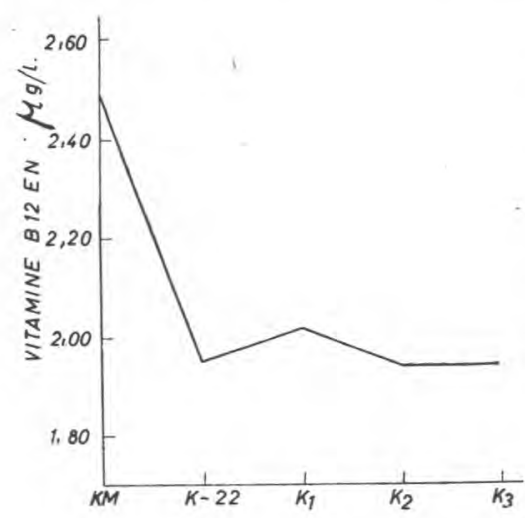

Fig. 1. - Les changements de teneur en vitamine B12 pendant la fabrication du Kéfir à partir de lait auquel on a ajouté les grains de Kéfir.

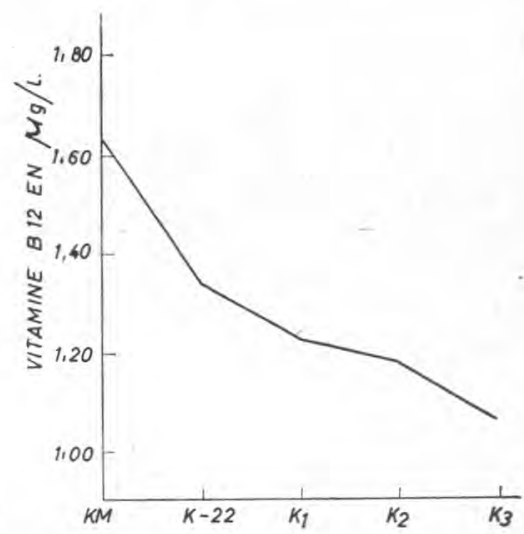

Fig. 2. - Les changements de teneur en vitamine B12 pendant la fabrication du Kéfir à partir de lait auquel on a ajouté la culturemère.

$\mathrm{KM}$ : le lait cuit.

K-22 : le Kéfir 18 heures après l'incubation à to de $22^{\circ} \mathrm{C}$.

$\mathrm{K} 1$ : le Kéfir après 1 jour à to de 8 à $10^{\circ} \mathrm{C}$.

K2 : le Kéfir après 2 jours à to de 8 à $10^{\circ} \mathrm{C}$.

$\mathrm{K} 3$ : le Kéfir après 3 jours à to de 8 à $10^{\circ} \mathrm{C}$.

En nous référant à cela, par les données du tableau I, on peut aussi voir que les pertes en vitamine B12 dans les Kéfirs faits de grains du Kéfir, sont beaucoup moindres que dans les Kéfirs faits de la culture-mère. Ainsi, dans le Kéfir fait des grains du Kéfir la perte en vitamine a été, en fin du troisième jour de 22,09 p. 100 tandis que dans le Kéfir fait de culture-mère elle était de 34,97 p. 100.

Dans l'explication des changements survenus dans la teneur en vitamine B12, il faut aller au fait qu'une flore microbienne assez complexe prend part à la fabrication et la maturation du Kéfir, cette flore microbienne étant composée de streptocoques lactiques, de leuconostocs, de lactobacilles et de levures (que fermentent ou ne fermentent pas le lactose). Pendant que certains microbes, comme les levures et certaines aroma-bactéries synthétisent la vitamine B12, les autres la dépensent. La tendance du changement de teneur en vitamine B12 pourra être par conséquent, le résultat d'une relation réciproque entre la création et la consommation de vitamine B12 par la microflore du Kéfir.

La différence constatée entre le kéfir fait des grains de Kéfir et celui fait de la culture-mère, quant aux pertes de vitamine B12, 
d'après les phases particulières de la fabrication et de la maturation, peut être expliquée par une représentation numérique inégale des composantes de la microflore. Dans le Kéfir fait du lait auquel on a ajouté les grains de Kéfir, la relation entre les levures et les autres microbes était, probablement, plus avantageuse que dans le Kéfir fait du lait auquel on a ajouté la culture-mère.

Quant à l'un et l'autre Kéfir, relativement la plus grande perte en vitamine $\mathrm{B} 12$, celle-ci était liée à la plus grande augmentation de l'acidité ; on pourra supposer que la perte de la vitamine dans cette phase est causée par l'évolution rapide des ferments lactiques qui dépensent la vitamine B12, e'est-à-dire que, pendant 18 heures d'incubation du lait inoculé à température de $22^{\circ} \mathrm{C}$ avec des bactéries du type Str. lactis et $\mathbf{S t}$. cremoris, viennent au jour les bactéries qui se distinguent par une plus grande multiplication que les autres microbes représentées dans les grains de Kéfir, ou dans la culturemère. Pour cette sorte de ferments lactiques, il est caractéristique qu'elle dépense la vitamine B12.

Comme dans la fabrication industrielle on applique, de plus en plus, la production du Kéfir du lait auquel on ajoute la culture-mère alors, les changements de teneur en vitamine B12 pendant la fabrication et la maturation du Kéfir réalisée de cette manière ont une plus grande importance pratique. Considérant que pendant la maturation de ce Kéfir, le taux de vitamine B12 se réduit sans cesse, il pourra être plus utile du point de vue de la diététique de consommer le Kéfir après un seul jour de maturation.

\section{Résumé}

Par les résultats obtenus de l'examen de la teneur en vitamine B12 pendant la fabrication et la maturation du Kéfir, on peut conclure comme suit :

Dans la fabrication et la maturation du Kéfir on a des pertes en vitamine B12. Dans le Kéfir fait du lait auquel on a ajouté les grains de kéfir, ces pertes atteignaient après trois jours de maturation en moyenne 22,09 p. 100 ; mais dans le kéfir fait du lait auquel on a ajouté la culture-mère elles étaient de 34,97 p. 100 par rapport au lait cuit.

La perte en vitamine B12, relativement la plus grande, se situe dans la première phase de la fabrication du Kéfir, c'est-à-dire, pendant l'incubation de 18 heures du lait inoculé par les grains ou la culture-mère à température de $22^{\circ} \mathrm{C}$.

Les raisons possibles du changement de teneur en vitamine B12 pendant la production du Kéfir sont discutées brièvement. 


\section{Summary}

From the results obtained after the survey of vitamin B12 content during the fabrication and ripening of kefir, the following conclusions can be drawn :

The fabrication and ripening of Kefir involve some losses in vitamin B12. In the product being made with milk in which Kefir fungi have been added, such losses reached an average proportion of 22,09 per cent after a three days ripening, but in the product being made with milk in which the mother culture has been added, the proportion of losses amounted to 34,97 per cent in comparison to the cooked milk.

The highest loss in vitamin B12, comparatively, occurs in the first stage of the fabrication of Kefir, i. e. during the 18 hours incubation of the milk inoculated with fungi or with mother culture at $22^{\circ} \mathrm{C}\left(72^{\circ} \mathrm{F}\right)$.

The possible reasons of the change in vitamin B12 content during the production of Kefir are briefly discussed.

\section{BIBLIOGRAPHIE}

[1] R. Karlin. R. C. R. Soc. Biol., 1961, 155, 1309.

[2] J. RASic I B. Panic. Archiv za Poljopr. nauke., 1961, 14, 43, 94.

[3] B. Randoin et J. Causeret. Le Lait, 1956, 36, 129.

[4] D. A. Callieri. Acta Chem. Scand., 1959, 13, 737.

[5] R. B. Davidov, L. E. Gulko, M. A. Ermakova. Osnovnie vitamini v moloke i molocnih produktah. Piscepromizdat. Moskva, 1956.

\section{NOTE SUR LA DÉGRADATION DES PROTIDES AU COURS DE LA MATURATION DU CAMEMBERT (1) par}

\section{J. LENOIR}

Le processus de maturation des fromages est dominé par trois grands phénomènes biochimiques : la fermentation du lactose, l'hydrolyse de la matière grasse et la dégradation des protides. Ces phénomènes, et plus particulièrement le dernier, modifient l'aspect, la texture et la consistance de la pâte ; simultanément, ils sont à l'origine du développement de la saveur du fromage.

(1) C. R. Acad. Agr., 1962, 48, no 3, 160. 